\title{
The Optimal Anatomical Position and Threshold Temperature of a Temperature Data Logger for Brace-Wearing Compliance in Patients with Scoliosis
}

Running head: Position and Threshold for Scoliosis Brace Logger

Keita Nakayama1), Toshiaki Kotani ${ }^{1)}$, Hiromi Kimura ${ }^{2)}$, Minako Osaki ${ }^{2)}$, Yuri

Ichikawa $^{3)}$, Tsuyoshi Sakuma1), Yasushi Iijima'), Kotaro Sakashita ${ }^{1)}$, Takahiro

Sunami ${ }^{4)}$, Tomoyuki Asada ${ }^{4)}$, Kosuke Sato ${ }^{4)}$, Tsutomu Akazawa ${ }^{5)}$, Shunji Kishida ${ }^{1)}$, Yu

Sasaki ${ }^{1)}$, Kazuhide Inage ${ }^{6}$, Yasuhiro Shiga ${ }^{6)}$, Shohei Minami ${ }^{1)}$, Seiji Ohtori'), Masao

Koda $^{4)}$, Masashi Yamazaki ${ }^{4}$

1) Department of Orthopedic Surgery, Seirei Sakura Citizen Hospital, Sakura, Japan

2) Department of Nursing, Seirei Sakura Citizen Hospital, Sakura, Japan

3) Medical Affairs Division, Seirei Sakura Citizen Hospital, Sakura, Japan

4) Department of Orthopedic Surgery, University of Tsukuba, Tsukuba, Japan

5) Department of Orthopedic Surgery, St. Marianna University, School of Medicine, Kawasaki, Japan

6) Department of Orthopedic Surgery, Chiba University, Graduate School of Medicine, Chiba, Japan

Corresponding author: Toshiaki Kotani

Department of Orthopedic Surgery, Seirei Sakura Citizen Hospital,

2-36-2 Ebaradai Sakura-shi, Chiba 285-8765, Japan

Tel: +81-43-486-1151

E-mail: tkotakotani@gmail.com

Conflicts of Interest: The authors declare that there are no relevant conflicts of interest.

Ethical Approval: No. 29-015, Institutional review board of Seirei Sakura Citizen Hospital.

Author Contributions: Keita Nakayama and Toshiaki Kotani wrote and prepared the manuscript, and all of the authors participated in the study design. All authors have 
read, reviewed, and approved the article.

Informed Consent: Informed consent was obtained from all participants in this study. 
1 The Optimal Anatomical Position and Threshold Temperature of a Temperature

2 Data Logger for Brace Wearing Compliance in Patients with Scoliosis

3

4

5

6 


\section{$1 \quad$ ABSTRACT}

3 Introduction: Although strict compliance with brace wearing is important for patients

4 with scoliosis, no study has analyzed the most ideal conditions for temperature logger

5 accuracy. We evaluated the optimal brace position and threshold temperature for the

6 logger and determined the reliability of its measurements in patients with scoliosis.

8 Methods: Five temperature loggers were embedded into holes generated at five different

9 brace positions (right scapula, right chest, left chest, lumbar, and abdomen) within the

10 brace. We compared measurement errors at each position using different threshold

11 temperatures to determine the ideal anatomical position and threshold temperature. Under

12 the ideal conditions determined, we calculated the reliability of the temperature logger

13 readings in three healthy participants.

15 Results: Measurement errors (i.e., differences between the actual and logger-recorded

16 brace wearing times) were the lowest at the $28^{\circ} \mathrm{C}$ and $30^{\circ} \mathrm{C}$ threshold temperatures when 
1 the logger was positioned at the left chest and at $30^{\circ} \mathrm{C}$ at the abdomen. Among these three

2 temperature/position combinations, we considered the abdomen to be the least affected

3 by the shape of the brace; thus, the placement of the temperature logger at the abdomen

4 using a threshold temperature of $30^{\circ} \mathrm{C}$ was the most ideal condition.

6 Conclusions: The placement of the temperature logger at the abdomen using a threshold

7 temperature of $30^{\circ} \mathrm{C}$ was the most ideal condition, with the reliability of the logger being

$897.9 \% \pm 70.9 \%$. This information might be useful for scoliosis management teams, and

9 this temperature logger provides a valuable clinical tool.

12 Key words: scoliosis, brace, compliance, temperature, logger 


\section{$1 \quad$ Main Text}

\section{INTRODUCTION}

4 effective for preventing spinal curvature progression. Patient compliance with brace

5 wearing affects the treatment outcome ${ }^{1,2)}$. However, because wearing a brace is stressful

6 and restricts daily movements, patients often stop wearing the brace and discontinue

7 treatment ${ }^{3-5}$. Moreover, patients may not report their accurate brace wearing time to

8 medical staff, purposively overestimating the time to appease the staff. Therefore, the

9 accurate measurement of brace wearing is crucial for patients with AIS.

11 monitors for brace treatment. Most electronic compliance monitors consist of a sensor,

12 clock, and battery-powered temperature logger to record sensor readings. These

13 monitors are generally categorized as temperature sensing ${ }^{1,6-8)}$, force sensing ${ }^{9-11)}$, or

14 both force and temperature sensing ${ }^{12)}$, of which temperature sensing is the most popular

15 monitoring method. These compliance monitors are placed in the brace and are set at a

16 threshold temperature to enable the differentiation of ambient temperature from the skin 
1 temperature of the patient ${ }^{1,7,12,13)}$. Wearing time is determined by a temperature logger

2 embedded in the brace programmed to $\log$ the time at a given temperature. A

3 temperature higher than the threshold indicates that the brace is being worn. However, no reports have assessed the optimal position within the brace or

5 threshold temperature of the temperature logger for obtaining accurate data. Moreover,

6 the reliability of the logger has not been accurately analyzed under different anatomical

7 positions/temperature conditions. Thus, the most ideal setting and reliability of

8 temperature loggers are unclear, although patient compliance with brace wearing is

9 associated with treatment outcome in patients with scoliosis ${ }^{1)}$.

11 threshold temperature of the temperature logger and evaluate the reliability of the logger

12 under the determined ideal conditions for the brace of the patient with AIS.

14 MATERIALS AND METHODS

15 Design of the Compliance Monitor 
1 a temperature sensor (Thermochron, KN Laboratories, Inc., Osaka, Japan). The

2 temperature logger weighs $3 \mathrm{~g}$, measures $17 \times 17 \times 6 \mathrm{~mm}$, and can store 2048 recordings

3 (Figure 1). The accuracy is $\pm 1^{\circ} \mathrm{C}\left(-40^{\circ} \mathrm{C}\right.$ to $\left.+80^{\circ} \mathrm{C}\right)$. The logger cannot be recharged, but

4 the battery lasts for 5 years or until the cumulative number of measurements exceeds one

5 million. The monitor can measure temperature at intervals of 1-255 minutes using a real

6 time clock. The monitor was set to record the temperature every 30 minutes for a total of

742 consecutive days. Data can be downloaded from the logger using an adapter that

8 connects to a computer.

9

10 First Study: Optimal Logger Position and Threshold Temperature for Logging

11 To determine the optimal position for temperature logging, the five temperature

12 loggers were embedded in holes cut in five different positions (right scapula, right chest,

13 left chest, lumbar, and abdomen) within the brace (Figure 2). The first study, conducted

14 in April 2018, aimed to measure the reliability of the electronic compliance monitor

15 placed at different positions within the brace. A nurse who works in the scoliosis clinic

16 (coauthor HK) was voluntarily recruited for this trial; she had no history of spinal 
1 deformity. She was asked to wear a custom-fitted thoracolumbosacral brace (Figure 2),

2 similar to that for orthosis for scoliosis, for 20 hours per day over 5 consecutive days.

The nurse recorded in a notebook the exact duration she wore the brace, and

4 this was defined as the "real time." Temperatures above the threshold indicate that the

5 brace is being worn, whereas temperatures below the threshold indicate that the brace

6 has been taken off. The duration at which the temperature exceeded the threshold was

7 calculated as the "log time." To determine the most appropriate temperature threshold,

8 thresholds of $28^{\circ} \mathrm{C}, 30^{\circ} \mathrm{C}$, and $32^{\circ} \mathrm{C}$ were compared. We calculated the percentage

9 difference as $(\log$ time - real time $) /$ real time $) \times 100$. The reliability under each

10 condition was calculated as $100-$ (absolute value of the percentage difference). We

11 then compared the reliabilities at each anatomical position and at three different

12 temperature thresholds to determine the ideal anatomical position and threshold.

14 Second Study: Reliability Test Using Three Volunteers 
1 the first study. Two nurses and one medical clerk who work at a scoliosis clinic

2 (coauthors HK, MO, and YI) were voluntarily recruited for this trial; none had a history

3 of spinal deformity. They were each asked to wear a custom-fitted thoracolumbosacral

4 brace over 20 hours a day for 7 consecutive days in August 2018. The tests were

5 performed during the hottest month (August) in this region where our facility was

6 located to validate the accuracy of the temperature monitor. We calculated the reliability

7 of the logger in each participant under the conditions established in the first study. The

8 institutional review board of our hospital approved this study protocol.

9

10 RESULTS

11 First Study

In the first study, the average "real time" that the brace was worn per day was

$1322: 18 \pm 0: 35$. The overall compliance rate calculated by dividing the real time by 24 (total

14 hours per day) was $92.9 \% \pm 2.4 \%$ (Table 1). The "log time" was calculated at the different

15 anatomical positions and temperature thresholds evaluated. As an example, Figure 3

16 shows a plot of temperature versus time recorded by the logger when positioned at the 
1 lumbar position and using a threshold temperature of $30^{\circ} \mathrm{C}$. Wearing time, indicated by

2 the double-headed arrows, was defined as the duration at which the temperature exceeded

$330^{\circ} \mathrm{C}$. When the brace is not being worn, the temperature remains at a low level, similar

4 to the ambient temperature. The dotted double-headed arrow indicates the duration at

5 which the brace was not being worn.

7 typical day. It was judged that the brace was being worn whenever the temperature

8 exceeded the designated threshold $\left(28^{\circ} \mathrm{C}, 30^{\circ} \mathrm{C}\right.$, and $\left.32^{\circ} \mathrm{C}\right)$. When this threshold was

9 changed, the wearing time (log time) also changed. When the threshold was set to $28^{\circ} \mathrm{C}$,

10 the $\log$ time was calculated as $22.5 \mathrm{~h}\left(93.8 \%\right.$ of the day). However, at the $32^{\circ} \mathrm{C}$ threshold,

11 the log time decreased to $19.5 \mathrm{~h}(81.3 \%)$. Thus, increasing the threshold temperature

12 resulted in a shorter log time. Calculating the log time from raw data obtained by the

13 logger took only a few minutes using Microsoft Excel (Microsoft Corp., Redmond, WA). 
1 respectively. Thus, the logger temperature was higher than the ambient temperature at all

2 times. Table 2 shows the percentage differences in the log versus real times for

4 different combinations between each of the five logger placement positions and each of

5 the three different temperature thresholds. The lowest percentage differences were

6 obtained when using the $28^{\circ} \mathrm{C}$ threshold at the left chest position, $30^{\circ} \mathrm{C}$ at the left chest,

7 and $30^{\circ} \mathrm{C}$ at the abdomen. Among these three position/temperature combinations, we

8 considered the abdomen to be the least affected by the shape of the brace; thus, the

9 placement of the logger on the abdomen at a threshold temperature of $30^{\circ} \mathrm{C}$ was the most

10 ideal combination.

12 Second Study In the second study, three participants wore braces with a logger installed on

14 their abdomen at a threshold temperature of $30^{\circ} \mathrm{C}$. These participants were asked to wear

15 the brace for as long as possible; however, because MO took the brace off when playing

16 volleyball, the wearing time was shorter for MO than for HK and YI. The average 
1 reliability among the three participants was $97.9 \% \pm 0.9 \%$. In August 2019, when the

2 second study was performed, the average highest and lowest temperatures of the day were

$3 \quad 32.4^{\circ} \mathrm{C}$ and $24.3^{\circ} \mathrm{C}$, respectively. That average ambient temperature was sometimes higher

4 than the threshold temperature.

\section{DISCUSSION}

We found that placing the logger on the abdomen at a threshold temperature of

$830^{\circ} \mathrm{C}$ was the most ideal condition. Under these parameters, we demonstrated that the

9 reliability of the logger was $97.9 \% \pm 0.9 \%$ according to the notes of the three participants

10 receiving scoliosis care. This is the first study to evaluate the ideal anatomical placement

11 and threshold temperature for temperature loggers used with scoliosis braces. threshold temperature of $30^{\circ} \mathrm{C}$ was the most ideal combination in terms of the difference

14 between the "real times" and "log times." Although temperature loggers were sensitive

15 in their place, no studies have compared the temperature logger readings among different

16 positions within the brace. In previous studies, loggers were primarily placed at the 
1 lumbar mold ${ }^{6,8,14-16)}$ and sometimes at the abdomen ${ }^{7}$. However, our results indicate that

2 the abdomen is one of the two most accurate positions for logger placement, perhaps

3 because the logger rarely loses contact with the subject's body at this site. In addition, an

4 abdominal logger has the advantage of not affecting the efficacy of the brace. Although

5 the measurement error of the logger at the left chest position, as one study described ${ }^{12)}$,

6 was as accurate as that at the abdomen, we consider placement at the left chest to be less

7 ideal, because the adjustment of pads in areas such as the left chest is sometimes necessary

8 during brace therapy.

Regarding the ideal threshold temperature, some clinical studies used sensor

10 thresholds between $28^{\circ} \mathrm{C}$ and $32^{\circ} \mathrm{C}^{1,7,14,17)}$. However, no previous studies have evaluated

11 the optimal threshold temperature. When the ambient temperature is high and the

12 threshold temperature is low, there is a risk of the logger mistakenly recognizing that the

13 brace is being worn. In contrast, if the threshold temperature is set too high, the logger

14 might not recognize the brace being worn. Among the $28^{\circ} \mathrm{C}, 30^{\circ} \mathrm{C}$, and $32^{\circ} \mathrm{C}$ thresholds,

15 we found $30^{\circ} \mathrm{C}$ to be optimal. 
1 study demonstrated the reliability of the temperature logger to be $97.9 \% \pm 0.9 \%$ by

2 comparing notes among the three participants engaged in scoliosis care. Takemitsu et al.

3 demonstrated a logger reliability of $97.6 \%$ by evaluating five volunteers for $1-3$ days ${ }^{6}$.

4 Our results were similar to those of Takemitsu et al. and Havey et al., who showed that

5 the mean difference between the data recorded by a logger and those manually recorded

6 in a diary was $0.9 \% \pm 2.2 \%{ }^{9}$. The error in their study was less than that in our study.

7 However, the study by Havey et al. differed from our study in that the wearing time of

8 the brace was only $1-8$ hours per day, which is shorter than the actual clinical use; in

9 addition, their investigation was conducted using four sensors at the same time ${ }^{9}$. In the

10 current study, we were able to achieve the same reliability using a single temperature

11 logger for approximately 20 hours/day. accurately record the total time of brace wearing in a notebook. In this study, nurses and

14 clerical staff involved in the treatment of scoliosis voluntarily wore the brace. Compared

15 with the volunteer participants in previous studies ${ }^{6,9,12)}$, our participants were more

16 credible because they understood the significance of the research better. Moreover, the 
1 experience of the three medical staff members of a scoliosis team wearing the brace for

2 several days might be useful for the care of future brace patients. If the ambient temperature is almost the same as the skin temperature, the

4 accuracy of the temperature logger decreases. In fact, in the first study, conducted in April,

5 the reliability of the logger in the most ideal setting was $99.8 \%$. On the other hand, in the

6 second study, conducted in August, the mean reliability of the three participants was

$7 \quad 97.9 \% \pm 0.9 \%$. As expected, the reliability was slightly lower in August because of the

8 higher ambient temperature; however, the error in August was only $2.1 \%$ or 30 minutes.

9 This error is considered to be clinically acceptable. Thus, the ambient temperature did not

10 greatly reduce the accuracy of the logger even in the hottest month. It may be because the

11 participants spent a lot of times indoors with air conditioning in the hottest time of

12 summer. Compared with force-sensing loggers ${ }^{9-12)}$, temperature sensing is simple to

13 perform, and the data easy to analyze with sufficient accuracy, and our results, which

14 showed a high reliability of $97.9 \% \pm 0.9 \%$ even during the hottest season, support the

15 clinical use of temperature sensing loggers. 
1 participants in this study was small. The participants had to wear the scoliosis brace most

2 of the day and record the exact wearing time for several days. Because this preliminary

3 trial could be a burden to healthy participants, we did not recruit additional participants.

4 Katz et al. also conducted a similar study in which as few as three subjects were included ${ }^{7)}$.

5 Second, the present study used a thoracolumbosacral brace only because this type of brace

6 is common. Different types of braces should be evaluated in future work. Third, the

7 present study was performed in healthy subjects only. We need to evaluate logger

8 function for a longer period in patients with AIS in a future study.

9

10 CONCLUSION

11 This study provides objective results confirming that the placement of the

12 temperature logger of an electronic compliance monitor at the abdominal position of the

13 brace using a threshold temperature of $30^{\circ} \mathrm{C}$ was the optimal condition for evaluating

14 brace compliance in patients with scoliosis. Under this condition, our results also

15 demonstrated that the reliability of the temperature logger was $97.9 \% \pm 0.9 \%$. This 
1 information can be useful for scoliosis management teams, and the temperature logger

2 of electronic compliance monitors provides a valuable clinical tool. 


\section{REFERENCES}

2 1. Weinstein SL, Dolan LA, Wright JG, et al. Effects of bracing in adolescents with

3 idiopathic scoliosis. N Engl J Med. 2013;369(16): 1512-21.

4 2. Hawary RE, Zaaroor-Regev D, Floman Y, et al. Brace treatment in adolescent

5 idiopathic scoliosis: risk factors for failure-a literature review. Spine J.

$6 \quad 2019 ; 19(12): 1917-25$.

7 3. Asada T, Kotani T, Nakayama K, et al. Japanese adaptation of the bad sobernheim

8 stress questionnaire-brace for patients with adolescent idiopathic scoliosis. J Orthop

$9 \quad$ Sci. 2019;24(6): 1010-4.

10 4. Misterska E, Glowacki M, Harasymczuk J. Polish adaptation of bad sobernheim

11 stress questionnaire-brace and bad sobernheim stress questionnaire-deformity. Eur

12 Spine J. 2009;18(12): 1911-9.

13 5. Piantoni L, Tello CA, Remondino RG, et al. Quality of life and patient satisfaction

14 in bracing treatment of adolescent idiopathic scoliosis. Scoliosis Spinal Disord. 
16 . Takemitsu M, Bowen JR, Rahman T, et al. Compliance monitoring of brace

2 treatment for patients with idiopathic scoliosis. Spine. 2004;29(18): 2070-4;

3 discussion 4.

4 7. Katz DE, Herring JA, Browne RH, et al. Brace wear control of curve progression in

5 adolescent idiopathic scoliosis. J Bone Joint Surg Am. 2010;92(6): 1343-52.

6 8. Rahman T, Sample W, Yorgova P, et al. Electronic monitoring of orthopedic brace compliance. J Child Orthop. 2015;9(5): 365-9.

8 9. Havey R, Gavin T, Patwardhan A, et al. A reliable and accurate method for measuring orthosis wearing time. Spine. 2002;27(2): 211-4.

10. Vandal S, Rivard CH, Bradet R. Measuring the compliance behavior of adolescents wearing orthopedic braces. Issues Compr Pediatric Nurs. 1999;22(2-3): 59-73.

12 11. Lou E, Hill D, Hedden D, et al. An objective measurement of brace usage for the treatment of adolescent idiopathic scoliosis. Med Eng Phys. 2011;33(3): 290-4.

14 12. Chalmers E, Lou E, Hill D, et al. An advanced compliance monitor for patients undergoing brace treatment for idiopathic scoliosis. Med Eng Phys. 2015;37(2): 
1 13. Rahman T, Bowen JR, Takemitsu M, et al. The association between brace compliance and outcome for patients with idiopathic scoliosis. J Pediatr Orthop. 2005;25(4): 420-2.

14. Helfenstein A, Lankes M, Ohlert K, et al. The objective determination of compliance in treatment of adolescent idiopathic scoliosis with spinal orthoses. Spine. 2006;31(3): 339-44.

15. Donzelli S, Zaina F, Martinez G, et al. Adolescents with idiopathic scoliosis and their parents have a positive attitude towards the Thermobrace monitor: results from a survey. Scoliosis Spinal Disord. 2017;12: 12. wear compliance. J Child Orthop. 2010;4(4): 343-7. spinal orthosis use for the treatment of adolescent idiopathic scoliosis. Spine. 
Spine Surgery and Related Research J- STAGE Advance Publication (J une 11, 2021)

(C) The J apanese Society for Spine Surgery and Related Research. CC BY- NC- ND 4.0 (https://creativecommons.org/licenses/by- nc- nd/4.0/). 


\section{FIGURES}

2

\section{Figure 1}

4 The temperature logger used in this study.

\section{$6 \quad$ Figure 2}

7 Custom-molded thoracolumbosacral orthosis instrument.

8 A, back; B, right; C, left; D, front.

9 Temperature loggers embedded into five holes cut at different positions within the brace.

10 (a, right scapula; b, right chest; c, left chest; d, lumbar; e, abdomen)

$12 \quad$ Figure 3

13 A typical temperature curve obtained by the temperature logger and our interpretation of

14 the data.

15 Double-headed arrows and the dotted double-headed arrow indicate the durations of

16 wearing and not wearing the brace, respectively. The dotted horizontal line represents the 
1 threshold temperature $\left(30^{\circ} \mathrm{C}\right)$.

2

\section{$3 \quad$ Figure 4}

4 The temperatures recorded by the temperature logger over a 24-hour day. The three

5 horizontal lines indicate the three threshold temperatures evaluated (solid line, $32^{\circ} \mathrm{C}$;

6 dashed line, $30^{\circ} \mathrm{C}$; dotted line, $28^{\circ} \mathrm{C}$ ). Temperatures exceeding the threshold were defined

7 as wearing time.

8

9

10

11 


\section{Figure 1}

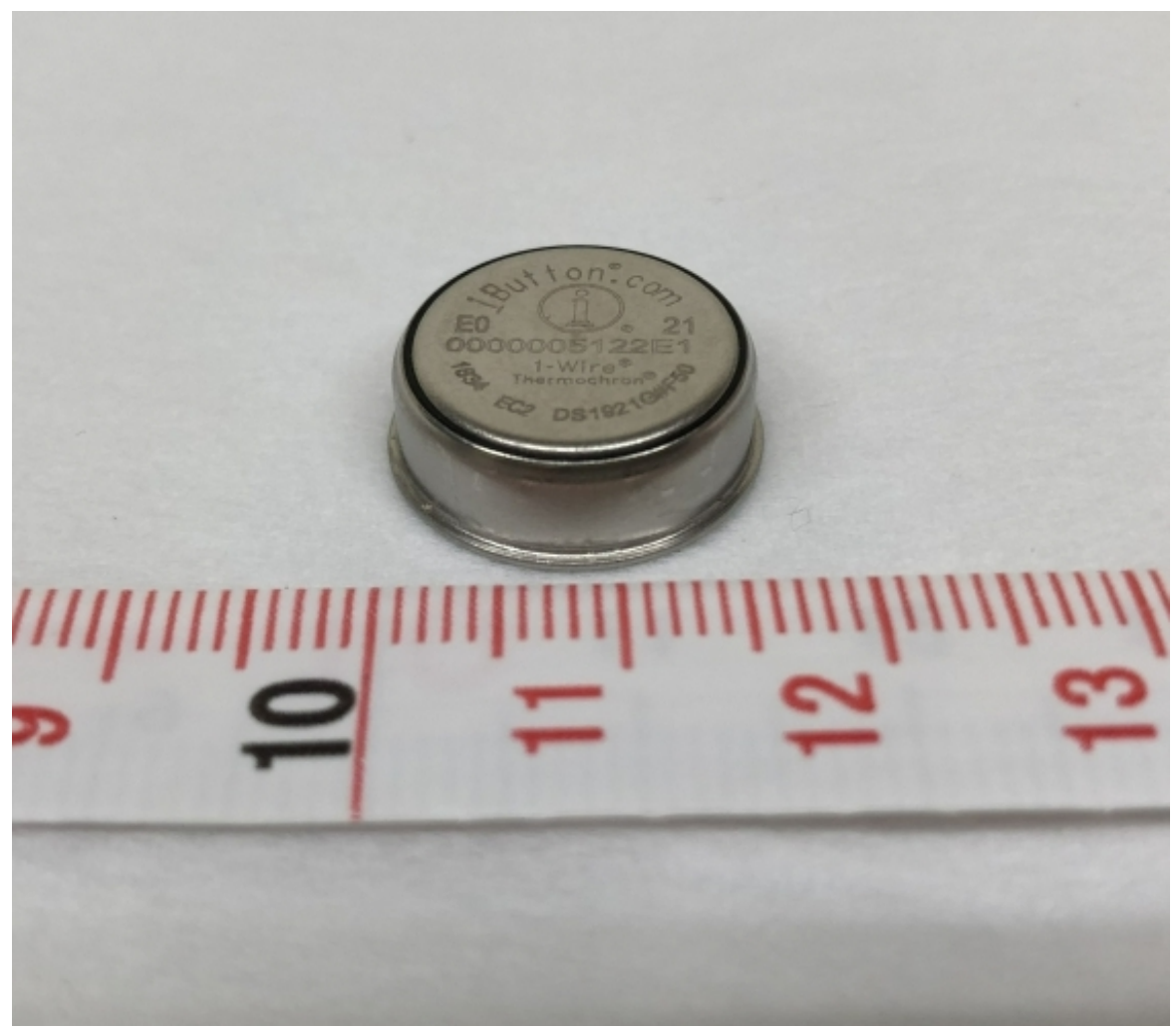


Spine Surgery and Related Research J-STAGE Advance Publication (J une 11, 2021)

(C) The J apanese Society for Spine Surgery and Related Research. CC BY- NC- ND 4.0 (https://creativecommons.org/licenses/by- nc- nd/4.0/).

Figure 2

A

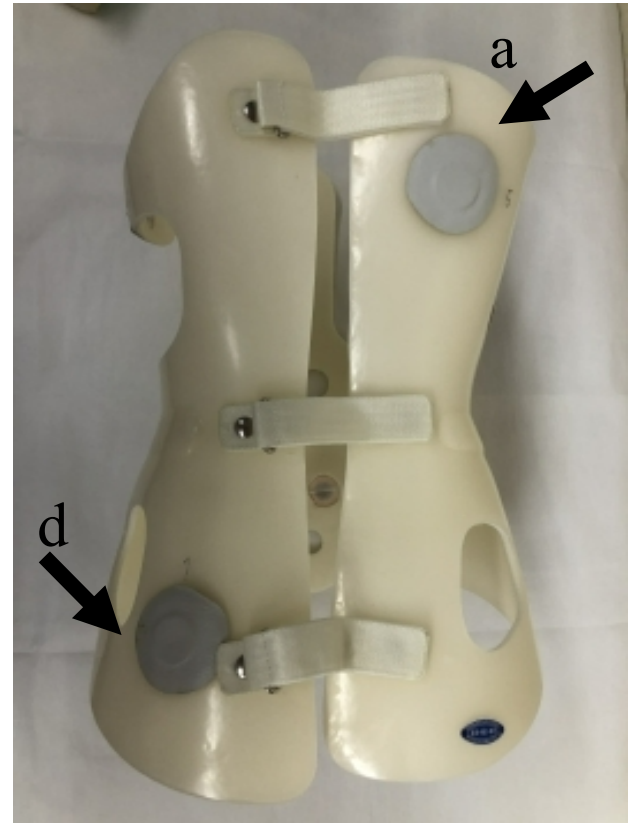

C

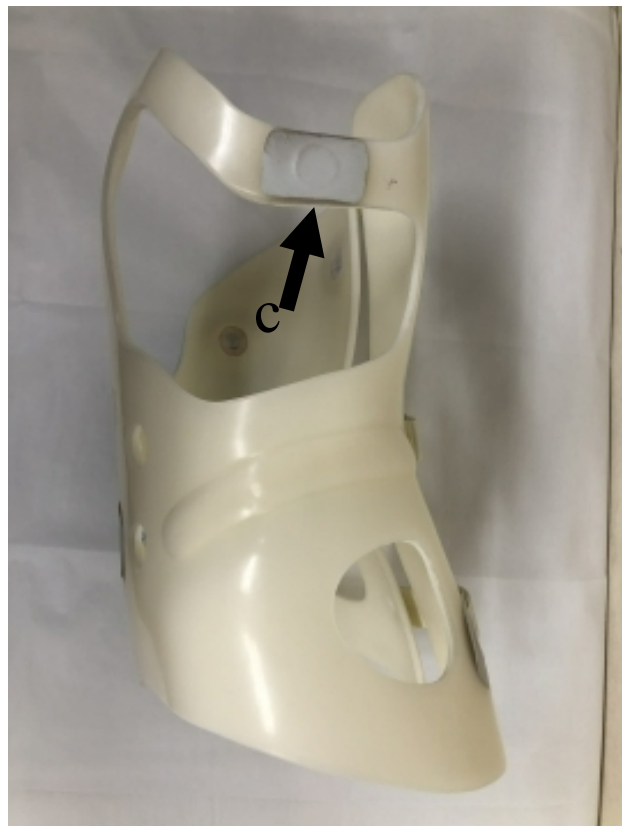

B

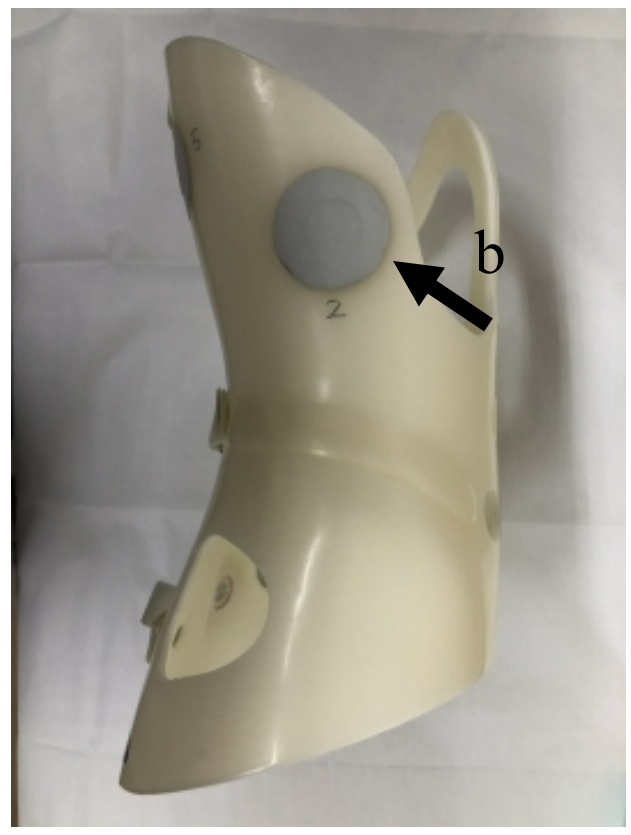

$\mathrm{D}$

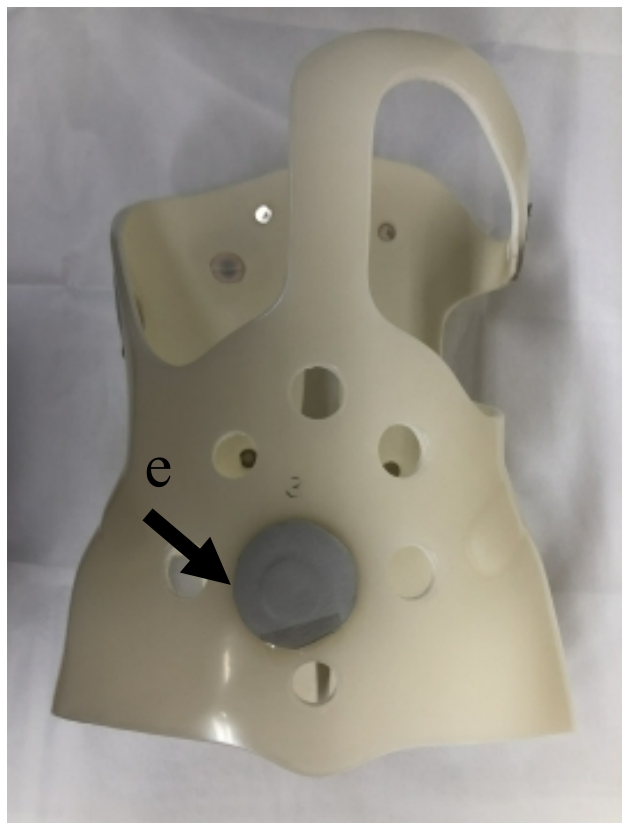




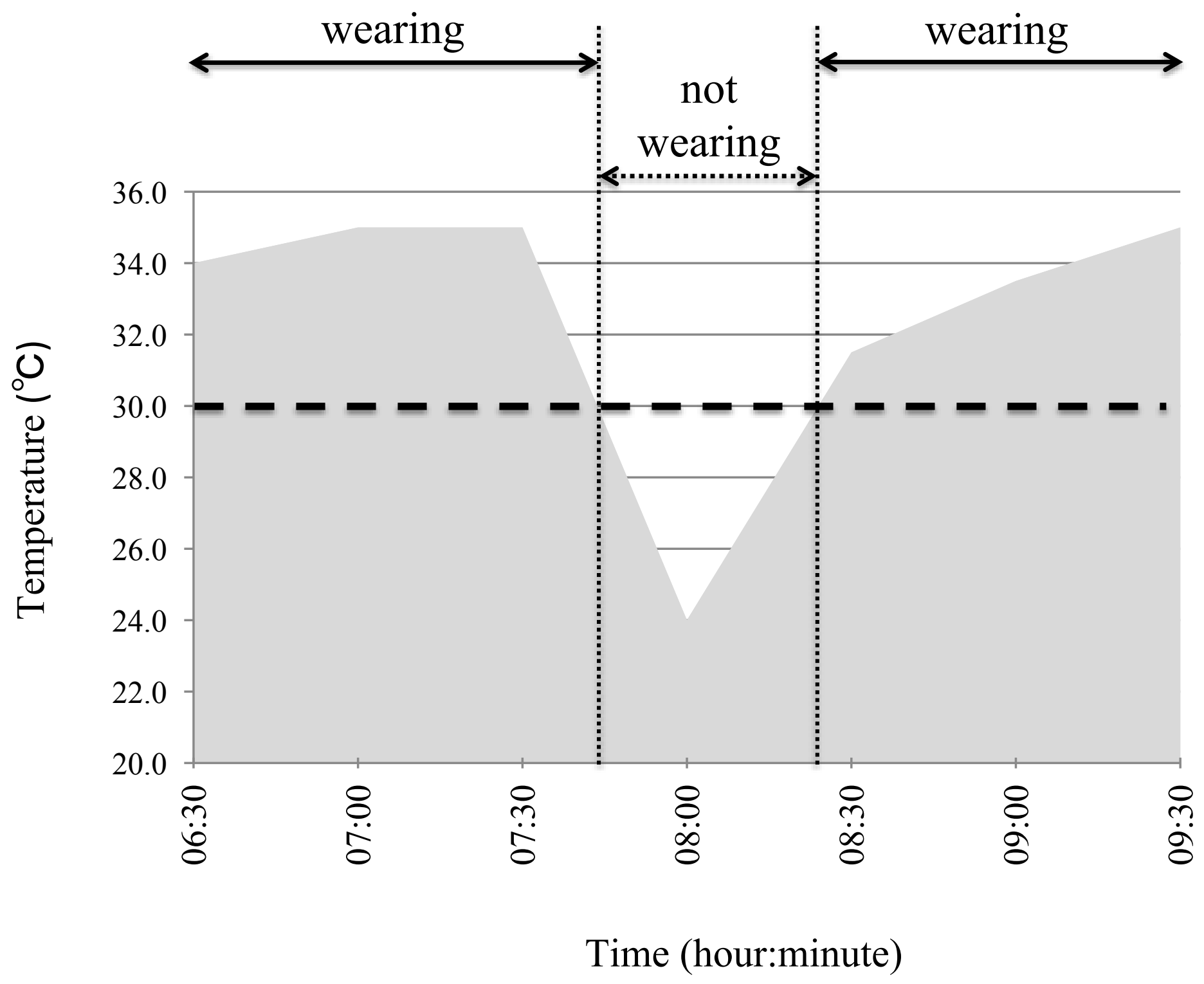


Figure 4

36.0

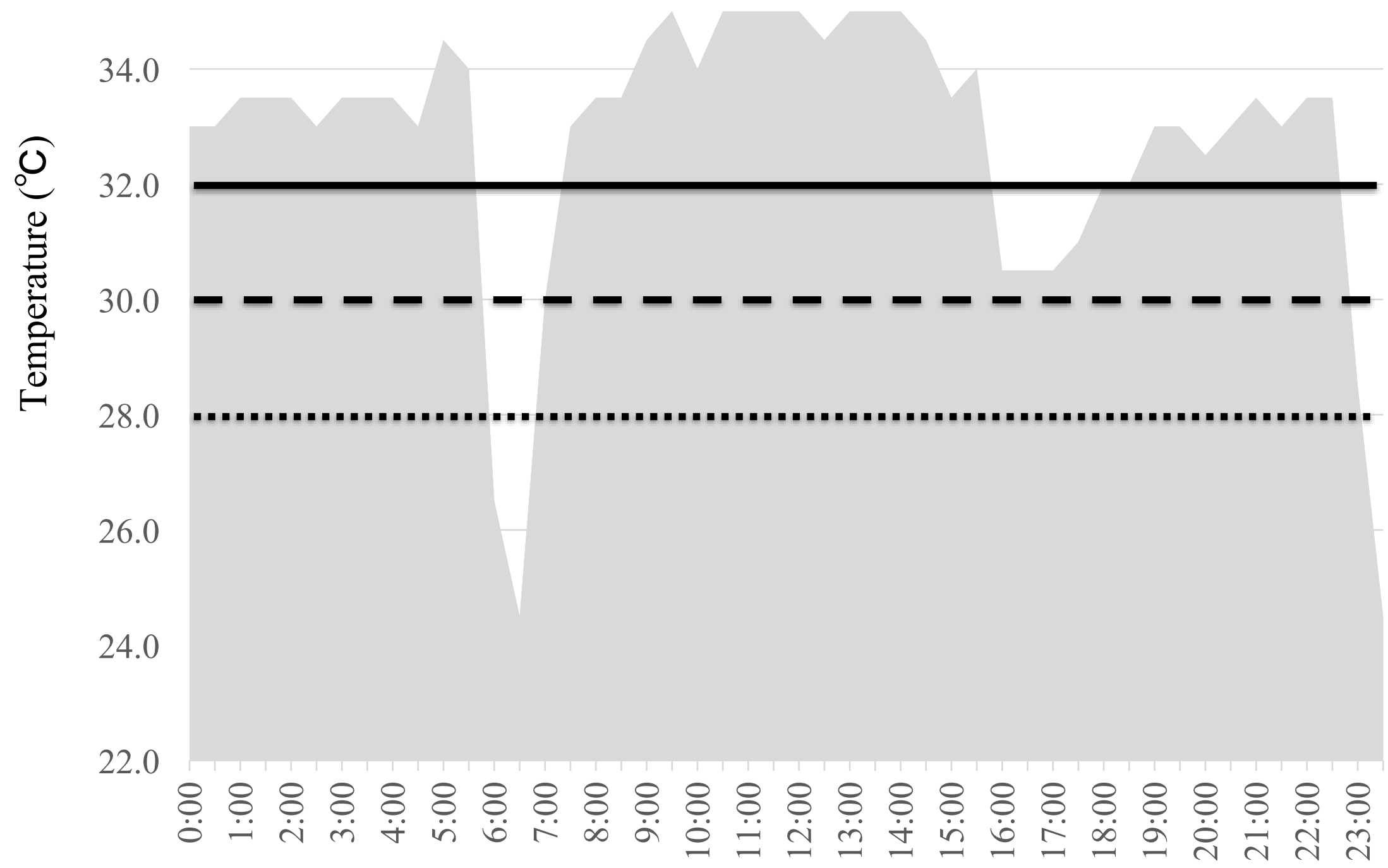

Time (hour:minute) 


\section{Tables}

\section{Table 1.}

Average "real time" per day of wearing the brace and the compliance rates over 5 consecutive days (first study)

\begin{tabular}{|c|c|c|}
\hline & $\begin{array}{c}\text { Real time } \\
\text { (recorded by notebook) } \\
\text { (hour:minute/day) }\end{array}$ & Compliance rate $(\%)$ \\
\hline Day 1 & $22: 45$ & 94.8 \\
\hline Day 2 & $21: 55$ & 91.3 \\
\hline Day 3 & $21: 20$ & 88.9 \\
\hline Day 4 & $22: 50$ & 95.1 \\
\hline Day 5 & $22: 40$ & 94.4 \\
\hline lean $\pm \mathrm{SD}$ & $22: 18 \pm 0: 35$ & $92.9 \pm 2.4$ \\
\hline
\end{tabular}

SD, standard deviation 
Table 2.

Percentage differences between the "real times" and "log times" at five different anatomical positions using three different threshold temperatures (first study)

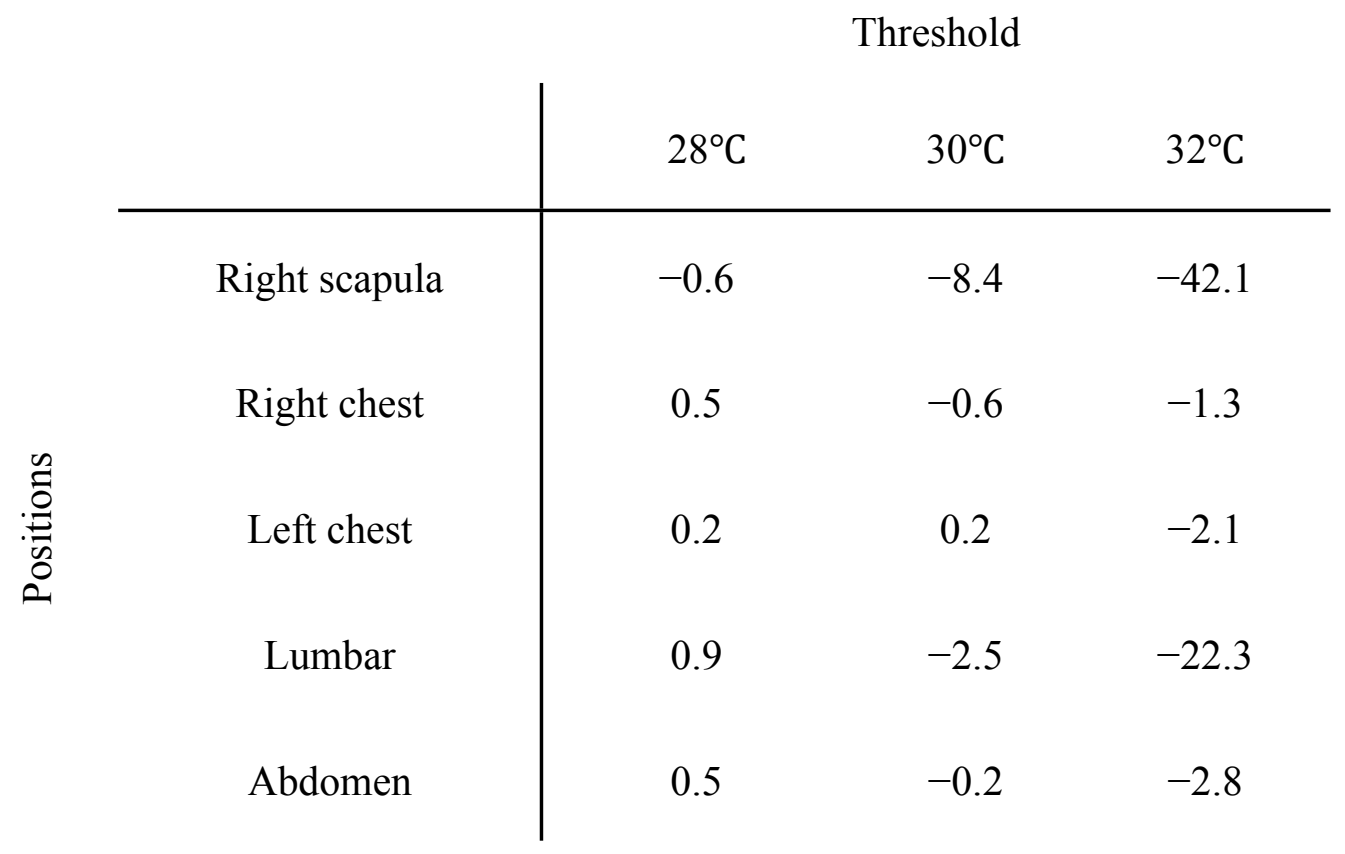




\section{Table 3.}

Data from the heathy volunteers in the second study showing the average time of

orthotic brace wear per day, recorded either in their notebooks or by the temperature

logger

\begin{tabular}{|c|c|c|c|c|}
\hline Subject & $\begin{array}{c}\text { Real time } \\
\text { (recorder by } \\
\text { notebook) } \\
\text { (hour:minute/day) }\end{array}$ & $\begin{array}{c}\text { Log time } \\
\text { (recorder by } \\
\text { logger) } \\
\text { (hour:minute/day) }\end{array}$ & $\begin{array}{c}\% \text { Difference } \\
(\%)\end{array}$ & $\begin{array}{c}\text { Reliability } \\
(\%)\end{array}$ \\
\hline HK & $22: 50$ & $23: 08$ & 1.36 & 98.6 \\
\hline MO & $19: 40$ & $19: 00$ & -3.38 & 96.6 \\
\hline YI & $22: 14$ & $21: 56$ & -1.37 & 98.6 \\
\hline Mean \pm SD & $21: 34 \pm 1: 22$ & $21: 21 \pm 1: 44$ & $2.04 \pm 0.95$ & $97.9 \pm 0.9$ \\
\hline
\end{tabular}

The mean and standard deviation of the percentage difference were calculated using the

absolute value of the percentage difference.

$\mathrm{SD}$, standard deviation 